\title{
FORCED NON-LINEAR VIBRATIONS OF A DAMPED SANDWICH BEAM
}

\author{
E. J. Kovac, JR $\dagger$ \\ Bell Telephone Laboratories, Baltimore, Maryland, U.S.A. \\ W. J. ANDERSON \\ Department of Aerospace Engineering, University of Michigan, \\ Michigan 48104, U.S.A. \\ R. A. SCOTT \\ Department of Engineering Mechanics, University of Michigan, \\ Michigan 48104, U.S.A.
}

(Received 5 October 1970)

\begin{abstract}
Forced, damped, non-linear, low-frequency flexural motions of a clamped-clamped sandwich beam with thin face sheets and a soft viscoelastic core are examined experimentally and theoretically. The theory employed neglects the extensional rigidity of the core and treats the face sheets as membranes. The non-linearity stems from axial stretching of the face sheets. Damping is taken into account by modeling the core as a Kelvin solid, with the material parameters used being obtained experimentally as functions of frequency and temperature. Theoretical frequency-amplitude relations are obtained using Galerkin's procedure and the method of harmonic balance. Results on fundamental natural frequencies, mode shapes, and stability are also presented. In the experiment, mechanical contact with the specimen was avoided by employing electromagnetic forcing and using a proximeter to measure displacements. Also, special attention was given to the interface bonds and to the reproduction, as close as possible, of clamped-clamped conditions. Agreement between the theoretical and experimental results is, in general, quite good.
\end{abstract}

\section{INTRODUCTION}

This paper is concerned with flexural vibrations of sandwich beams with clamped ends. In particular, non-linear axial stretching and constrained viscoelastic damping are considered. These topics have received considerable attention in recent years.

Extensive studies regarding the effect of non-linear axial stretching on the motion of homogeneous beams have been done. For an account of the early work the reader is referred to an article by Eisley [1]. Some other recent papers in the area should also be noted [2-6]. None of the above authors considered damping, and, indeed, for homogeneous metal beams, damping is negligible. However, for other systems, including many of the new composite structures, damping is not an insignificant factor. In particular, it is not negligible for structures which involve viscoelastic materials. Such materials have long been recognized as excellent vibration dampers. Viscoelastic coatings and tapes have frequently been applied to help control flexural vibrations. If a viscoelastic layer is in turn constrained by a stiff backing layer, even greater damping can be introduced. This sandwich construction induces shearing motion in the viscoelastic layer, which is a very effective damping mechanism. Analytically, damping of such structures has usually been treated by the complex moduli approach (see Ruzika [7]). 
Work on the flexural vibrations of sandwich beams seems to have focussed on three broad areas: namely, free, damped, linear motions; forced, damped, linear motions; and free, undamped, non-linear motions. Extensive theoretical results in the area, for beams and plates, have been presented by $\mathrm{Yu}$. A theory for linear, undamped motions was presented in reference [8], and a simplified version, suited for low-frequency motions of beams or plates with thin face sheets and soft cores, was given in reference [9]. In [8] and [9], free vibration problems were considered, the effect of damping being included in a later work [10], by means of the complex moduli approach. Yu [11] also developed theories for non-linear undamped motions and applied them to free vibration problems. His work on the free, undamped, non-linear vibrations of sandwich plates and shells, using a variational framework as a vehicle for approximation, should also be noted (see [12]). However, damped, non-linear motions were not treated. Also, no experimental support of the non-linear theories has been given.

Experiments involving the linear theories have been conducted. Nicholas and Heller [13] tried to determine the complex shear moduli of viscoelastic materials by using them as cores of sandwich beams. They considered the free, linear, vibrations of cantilever beams, which were constructed of a polyurethane core [shear modulus $G=1300 \mathrm{lb} / \mathrm{in}^{2}\left(8963 \mathrm{kN} / \mathrm{m}^{2}\right)$ ] and steel shim stock facings. Comparison between theory and experiment revealed scatter on the order of $\pm 15 \%$ and greater. Jones, Salerno and Savacchio [14] also reported experimental results for sandwich beams with viscoelastic cores. They considered two types of core material: namely, a very soft elastomer, butyl rubber $\left[G=700 \mathrm{lb} / \mathrm{in}^{2}\left(4826 \mathrm{kN} / \mathrm{m}^{2}\right)\right]$ and a stiffer thermoplastic, polyvinyl chloride $\left[G=10000 \mathrm{lb} / \mathrm{in}^{2}\left(68978 \mathrm{kN} / \mathrm{m}^{2}\right)\right]$. Theoretical and experimental natural frequencies showed discrepancies of the order of $10 \%$ for the thermoplastic specimens, and of the order of $90 \%$ for the soft elastomer materials. Damping factor discrepancies were even more pronounced. Finally, experimental results were also given by Agbasier and Grootenhuis [15]. They considered cantilever sandwich beams, using relatively stiff polyvinyl chloride as a core. Comparison of the theoretical (linear) and experimental frequency amplitude relations showed discrepancies of the order of $10 \%$ and less.

In the light of the above survey, it was decided that more work was required on sandwich beams with soft elastomer cores. Forced vibrations of such structures required further investigation, and the damped response needed to be extended to the non-linear case. The work presented here involves a theoretical and experimental investigation of the forced, damped, non-linear, low-frequency, flexural vibrations of clamped-clamped sandwich beams with soft elastomer cores. The non-linearity arises from axial stretching of the face sheets, and the damping stems from the shear deformation of the core. The governing equations which are employed are extensions of Yu's [11] simplified theory in that damping is injected by modelling the core material as a Kelvin solid. Frequency-amplitude relations are obtained from the theory by means of Galerkin's procedure and the method of harmonic balance. Stability is investigated and found to be governed by a third-order ordinary differential equation with time-dependent coefficients, which is analyzed by analog computer techniques. The theoretical predictions and experimental data for several specimens are compared with regard to fundamental natural frequencies, frequency-amplitude relations, and mode shapes.

\section{GOVERNING EQUATIONS}

The coordinates used are $x$ and $z$, where $x$ is along the mid-surface of the undeformed beam and $z$ is transverse to the thickness. The origin of coordinates is at the left end of the beam and $z$ is positive downwards.

Patterned after Nicholas and Heller [13] and Yu [11], it is assumed that (i) the core experiences predominantly shear deformations and possesses no extensional rigidity; (ii) the face 
sheets have no flexural rigidity and undergo no shear deformation; (iii) the transverse displacements for the top face, core, and bottom face are the same for the same value of $x$, i.e.

$$
w_{\mathrm{f}}^{\mathrm{l}}=w_{\mathrm{c}}=w_{\mathrm{f}}^{\mathrm{b}}=w,
$$

where here, and subsequently, f and $c$ denote face and core, respectively, and $t$ and $b$ designate top and bottom, respectively; (iv) when an angle $\psi$ is defined such that the total shearing deformation experienced by the core is given by $\psi+\theta$, where

$$
\theta=w^{\prime}
$$

the prime denoting differentiation with respect to $x$, the axial displacements are

$$
\begin{gathered}
u_{\mathrm{f}}^{\mathrm{t}}=-\frac{h}{2} \psi, \\
u_{\mathrm{c}}=z \psi, \\
u_{\mathrm{f}}^{\mathrm{b}}=\frac{h}{2} \psi,
\end{gathered}
$$

where $h$ denotes the core thickness.

Shown in Figure 1 are the various stress components, stress resultants, etc., consistent with the above assumptions, that act on elements of the face sheets and core. $N$ denotes a normal force due to bending, $S$ the axial stretching force (assumed independent of $x$ ), $\sigma$ an interface normal stress, $\tau$ an interface shear stress, $M$ a bending moment, and $Q$ a shear force. The subscript $s$ designates a surface quantity. Equilibrium of the various elements leads to six equations. With the assumption of perfect bonds, i.e.

$$
\begin{array}{rlrl}
\sigma_{\mathrm{f}}^{\mathrm{t}}=\sigma_{\mathrm{c}}^{\mathrm{t}}, & & \tau_{\mathrm{f}}^{\mathrm{t}}=\tau_{\mathrm{c}}^{\mathrm{t}}, \\
\sigma_{\mathrm{f}}^{\mathrm{b}}=\sigma_{\mathrm{c}}^{\mathrm{b}}, & \tau_{\mathrm{f}}^{\mathrm{b}}=\tau_{\mathrm{c}}^{\mathrm{b}},
\end{array}
$$

and when only external loads in the transverse direction are considered, i.e.

$$
\begin{gathered}
\sigma_{\mathrm{s}}^{\mathrm{t}}-\sigma_{\mathrm{s}}^{\mathrm{b}}=a(x) q(t), \\
\tau_{\mathrm{s}}^{\mathrm{t}}=0, \quad \tau_{\mathrm{s}}^{\mathrm{b}}=0,
\end{gathered}
$$

where $a$ and $q$ denote the spatial and time dependence of the load, respectively, the six equations can be reduced, after some algebraic manipulation, and use of equations (1), (3), and (5), to

$$
\begin{gathered}
\left(N^{\mathrm{b}}+N^{\mathrm{t}^{\prime}}\right) \theta+\left(N^{\mathrm{b}}+N^{\mathrm{t}}+2 S\right) \theta^{\prime}+Q_{\mathrm{c}}^{\prime}+a(x) q(t)=\left(\rho_{\mathrm{c}} A_{\mathrm{c}}+2 \rho_{\mathrm{f}} A_{\mathrm{f}}\right) \ddot{w} \\
M_{\mathrm{c}}^{\prime}-Q_{\mathrm{c}}+\frac{h}{2}\left(N^{\mathrm{b}^{\prime}}-N^{\mathrm{t}^{\prime}}\right)=\left(\rho_{\mathrm{c}} I_{\mathrm{c}}+\frac{1}{2} \rho_{\mathrm{f}} A_{\mathrm{f}} h^{2}\right) \bar{\psi}
\end{gathered}
$$

where $\rho$ denotes density, $A$ a cross-sectional area, $I_{\mathrm{c}}$ the area moment of inertia of the core about the $y$ axis, and the dot differentiation with respect to time.

Within the framework of the present model, the constitutive laws for the face sheets are, with the use of equations (3) and (5),

$$
\begin{aligned}
& N^{\mathrm{t}}=-\frac{1}{2} E_{\mathrm{f}} A_{\mathrm{f}} h \psi^{\prime}, \\
& N^{\mathrm{b}}=\frac{1}{2} E_{\mathrm{f}} A_{\mathrm{f}} h \psi^{\prime},
\end{aligned}
$$

where $E$ is Young's modulus. The axial stretching force is given by

$$
S=\frac{E_{\mathrm{f}} A_{\mathrm{f}}}{2 l} \int_{0}^{l}\left(w^{\prime}\right)^{2} \mathrm{~d} x,
$$


where $l$ is the beam length. If damping were neglected, the core constitutive equations would be

$$
\begin{gathered}
M_{\mathrm{c}}=E_{\mathrm{c}} I_{\mathrm{c}} \psi^{\prime}, \\
Q_{\mathrm{c}}=k^{2} A_{\mathrm{c}} G_{\mathrm{c}}\left(\psi+w^{\prime}\right),
\end{gathered}
$$

where $k$ is the so-called shear coefficient. The value for $k$ is determined by the method given by $\mathrm{Yu}$ [9]. In the present problem, damping cannot be ignored, and equations (14) and (15) have to be modified. Even though the ultimate concern here is with harmonic motions, the complex moduli approach is not a very convenient way to handle damping in a non-linear system. One

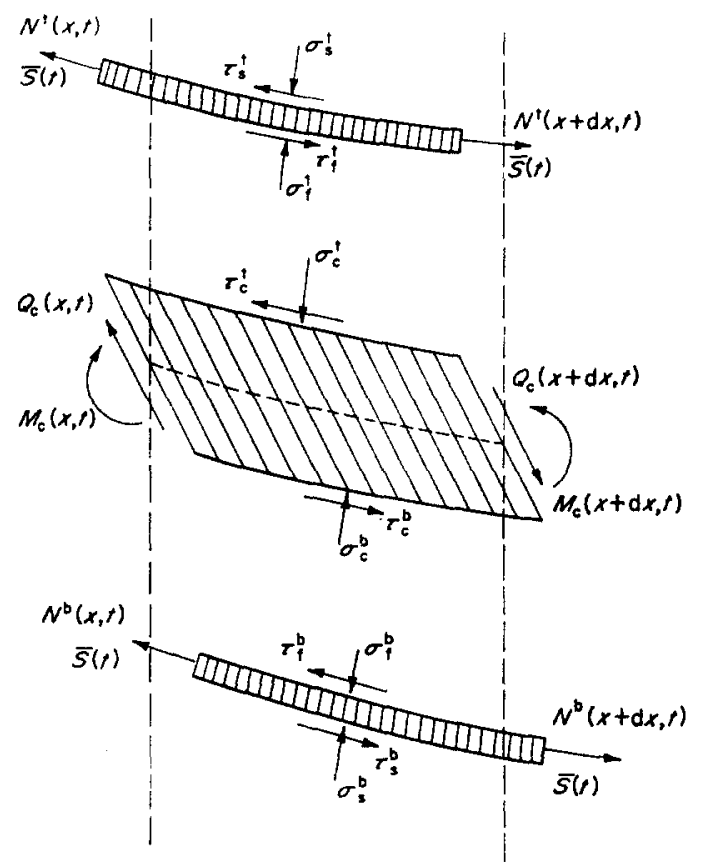

Figure 1. Equilibration diagram.

reason for this is that any assumed exponential behavior does not cancel out of the equations of motion. Moreover, arbitrary time-dependence is desired at this stage in order to develop equations governing stability. The method employed here, which is somewhat analogous to the complex moduli approach, is to assume that the core behaves as a Kelvin solid, i.e.

$$
\begin{aligned}
& \sigma_{\mathrm{c}}=E_{\mathrm{c}} \epsilon_{\mathrm{c}}+\zeta_{\mathrm{c}} \dot{\epsilon}_{\mathrm{c}}, \\
& \tau_{\mathrm{c}}=G_{\mathrm{c}} \gamma_{\mathrm{c}}+\eta_{\mathrm{c}} \dot{\gamma}_{\mathrm{c}},
\end{aligned}
$$

where $\epsilon$ is a normal strain, $\gamma$ a shear strain, and $\zeta_{c}, \eta_{c}$ are additional material parameters. Note that similar behavior in tension and shear is assumed. That this relatively simple model is adequate is borne out by the subsequent results. The Kelvin parameters in general vary with frequency and temperature. Much of the experimental data on material behavior is given in terms of complex moduli and a relationship between the two is desirable. This is readily achieved, as the following discussion shows.

It can be established that, for harmonic motion of frequency $\omega$,

$$
\begin{gathered}
E_{\mathrm{c}}=E_{1}, \\
\zeta_{\mathrm{c}}=E_{2} / \omega,
\end{gathered}
$$


where $E_{1}$ is the storage modulus, and $E_{2}$ is the damping modulus. The loss factor is given by

$$
\tan \delta=E_{2} / E_{1},
$$

and the analogous results for shear behavior are

$$
\begin{gathered}
G_{\mathrm{c}}=G_{1}, \\
\eta_{\mathrm{c}}=G_{2} / \omega, \\
\tan \delta_{\mathrm{sh}}=G_{2} / G_{1} .
\end{gathered}
$$

Since soft elastomers are essentially incompressible, Poisson's ratio $\nu_{\mathrm{c}}$ can be taken equal to $1 / 2$; then, from equation (18),

$$
G_{\mathrm{c}}=E_{\mathrm{c}} / 2\left(1+\nu_{\mathrm{c}}\right)=E_{1} / 3 \text {. }
$$

Also, it has been found experimentally that for most rubber-like materials, the loss factor is about the same in tension and shear, i.e. $\delta=\delta_{\mathrm{sh}}$. Hence, with the use of equations (20), (21), (22), and (23),

$$
\eta_{\mathrm{c}}=\left(E_{1} / 3 \omega\right) \tan \delta
$$

Thus the Kelvin moduli can be obtained from equations (18),(19), (24), and (25) if the complex moduli are known, an item that will be discussed later.

With the use of equations (16) and (17) it can be shown that, with the inclusion of damping, equations (14) and (15) become

$$
\begin{gathered}
M_{\mathrm{c}}=E_{\mathrm{c}} I_{\mathrm{c}} \psi^{\prime}+\zeta_{\mathrm{c}} I_{\mathrm{c}} \psi^{\prime} \\
Q_{\mathrm{c}}=k^{2} A_{\mathrm{c}} G_{\mathrm{c}}\left(\psi+w^{\prime}\right)+k^{2} A_{\mathrm{c}} \eta_{\mathrm{c}}\left(\psi+\dot{w}^{\prime}\right) .
\end{gathered}
$$

When equations (11), (12), (13), (26) and (27) are inserted into equations (9) and (10), the following equations of motion are obtained:

$$
\begin{gathered}
\left(B+E_{\mathrm{c}} I_{\mathrm{c}}\right) \psi^{\prime \prime}-K\left(\psi+w^{\prime}\right)-\bar{K}\left(\psi+\dot{w}^{\prime}\right)+\rho_{\mathrm{c}} I_{\mathrm{c}} \psi^{\prime \prime}=\rho_{\mathrm{c}} I_{\mathrm{c}}\left(1+2 \rho_{\mathrm{f}} f / \rho_{\mathrm{c}} h\right) \bar{\psi} \\
K\left(\psi^{\prime}+w^{\prime \prime}\right)+\bar{K}\left(\psi^{\prime}+\dot{w}^{\prime \prime}\right)+\left[L \int_{0}^{l}\left(w^{\prime}\right)^{2} \mathrm{~d} x\right] w^{\prime \prime}+a(x) \quad q(t)=m \ddot{w}
\end{gathered}
$$

where

$$
\begin{aligned}
& B=\frac{1}{2} E_{\mathrm{f}} A_{\mathrm{f}} h^{2}, \quad K=k^{2} G_{\mathrm{c}} A_{\mathrm{c}}, \\
& \bar{K}=k^{2} \eta_{\mathrm{c}} A_{\mathrm{c}}, \quad L=E_{\mathrm{f}} A_{\mathrm{f}} / l, \\
& m=\rho_{\mathrm{c}} A_{\mathrm{c}}+2 \rho_{\mathrm{f}} A_{\mathrm{f}},
\end{aligned}
$$

and $f$ is the face sheet thickness. In the sequel, the terms involving $I_{c}$, which are considerably smaller than the other terms which arise, are deleted.

Equations (28) and (29) are an extension of Yu's [11] simplified theory in that damping has been injected by means of modeling the core material as a Kelvin solid. These equations hinge on the face sheets being much thinner than the core. Yu [9] has stated that, for the lineary theory to be accurate, the following inequalities must hold:

$$
\begin{gathered}
R_{1}=2 f / h \ll 1, \\
R_{2}=\pi^{2} G_{\mathrm{f}} f^{3}(h+2 f) / 3\left(1-v_{\mathrm{g}}\right) G_{\mathrm{c}} h^{2} k^{2} l^{2} \ll 1 .
\end{gathered}
$$

He also stated that if the satisfaction of these inequalities is marginal, the fundamental branch frequency $\omega_{\mathrm{st}}$ of an infinite undamped beam, as calculated from the linear version of the current theory, could be improved upon by using the expression

$$
\omega_{\mathrm{m}}=\omega_{\mathrm{si}}\left(1+R_{1}+R_{2}\right)^{1 / 2}
$$


where $\omega_{m}$ denotes the improved estimate. In certain cases, equations (30), (31) and (32) are a valuable adjoint to the central theory.

Within the framework of the present theory, the boundary conditions for clamped ends are

$$
\begin{array}{ll}
w(0, t)=0, & w(l, t)=0, \\
\psi(0, t)=0, & \psi(l, t)=0 .
\end{array}
$$

In the following section, several aspects of the theoretical response to a time harmonic external load are developed. In particular, items such as frequency-amplitude relations, fundamental natural frequencies, mode shapes, and stability are studied. A major purpose of the paper is to test the adequacy of the present model by comparing the theoretical results with experimental ones.

\section{THEORETICAL PROCEDURE}

An important quality of a theory is the accuracy of its predictions regarding the relationship between frequency of an applied load and the amplitude of the corresponding response. Since equation (29) is non-linear, exact frequency-amplitude relations cannot be obtained, and approximate techniques must be employed. Here, to reduce equations (28) and (29) to ordinary differential equations, Galerkin's method is used. In broad outline, Galerkin's method, and the particular version employed here, are as follows. The response is assumed to be of the form

$$
\begin{aligned}
& w(x, t)=W \xi(x) \tau(t), \\
& \psi(x, t)=\Psi \mu(x) T(t),
\end{aligned}
$$

where $W$ and $\Psi$ are amplitude factors (constants) and the functions $\xi$ and $\mu$ are approximate, but satisfy the boundary conditions, equations (33) and (34). Usually $\xi$ and $\mu$ are taken as linear space modes, combinations of linear space modes, or approximations to the space modes. Note that in anticipation of at least a phase difference, different displacement and shear time-dependencies are used. Due to the approximate nature of $\xi$ and $\mu$, errors arise when equations (35) and (36) are substituted into equations (28) and (29). These errors are minimized if integrals, over the spatial interval, of their products with suitable weight functions are required to be zero. Frequently, the weight functions employed are $\xi$ and $\mu$.

The approximations chosen for the problem at hand are

$$
\begin{gathered}
\xi(x)=\frac{x^{2}}{l^{2}}-\frac{2 x^{3}}{l^{3}}+\frac{x^{4}}{l^{4}}, \\
\mu(x)=\frac{\mathrm{d} \xi}{\mathrm{d} x}=\frac{2 x}{l^{2}}-\frac{6 x^{2}}{l^{3}}+\frac{4 x^{3}}{l^{4}} .
\end{gathered}
$$

These expressions satisfy the theoretical boundary conditions. In addition, it follows from equations (37) and (38) that $w^{\prime}(0)=0$ and $w^{\prime}(l)=0$. This choice of function closely models the experimental conditions employed later. Furthermore, as will emerge, the undamped natural frequencies that stem from these assumed functions are almost identical with the exact solution of the linear governing equations given by Nicholas and Heller [13] for the boundary conditions, equations (33) and (34).

When equations (35), (36), (37), and (38) are inserted into equation (28), and the result is multiplied by $\xi^{\prime}(x)$, with integration from 0 to $l$,

$$
A_{1} \dot{\tau}+A_{2} T+B_{1} \tau+B_{2} T=0
$$


where

$$
\begin{aligned}
A_{1} & =2 \bar{K} b W / l, & A_{2} & =\bar{K} b \Psi_{1}, \\
B_{1} & =2 K b W / l, & B_{2} & =(K \bar{b}-B \tilde{a}) \Psi_{1}, \\
\bar{a} & =-1 / 5 l, & \bar{b} & =l / 210, \quad \Psi_{1}=2 \Psi / l .
\end{aligned}
$$

When equations (35), (36), (37), and (38) are substituted into equation (29) and the result is multiplied by $\xi(x)$, with integration from 0 to $l$,

where

$$
\ddot{\tau}+\gamma_{1} \dot{\tau}+\gamma_{2} \dot{T}+\alpha_{1} \tau+\alpha_{2} \tau+\beta \tau^{3}=\zeta q(t)
$$

$$
\begin{aligned}
\alpha_{1} & =-2 K \bar{a}_{1} / m \bar{a}_{3} l, & & \gamma_{1}=(\bar{K} / K) \alpha_{1}, \\
\alpha_{2} & =-K \bar{a}_{1} \Psi_{1} / m \bar{a}_{3} W, & & \gamma_{2}=(\bar{K} / K) \alpha_{2}, \\
\beta & =-\left(2 L \bar{b}_{1} \bar{a}_{1} / m \tilde{a}_{3} l\right) W^{2} \equiv \bar{B}_{1} W^{2}, & & \\
\bar{\zeta} & =\left(\bar{a}_{4} / m \bar{a}_{3}\right)(1 / W) \equiv \bar{C}_{1} / W, & & \\
\tilde{a}_{1} & =-1 / 105, & & \bar{b}_{1}=2 / 105 l, \\
\tilde{a}_{3} & =l / 630, & & \bar{a}_{4}=0.032 .
\end{aligned}
$$

In equations (39) and (40), the particular spatial dependence of the load used is given by

$$
a(x)= \begin{cases}0, & 0<x<l / 2-1 / 4 \\ 1, & l / 2-1 / 4<x<l / 2+1 / 4, \quad l=10^{\prime \prime}, \\ 0, & l / 2+1 / 4<x<l\end{cases}
$$

a choice which approximates the experimental conditions to be described later.

As will emerge, the time-dependence of the external load employed is of the form $(1+$ $\cos \omega t)$. The effect of the constant term has been shown by Bennett and Eisley [6] to be small for the case of a homogeneous beam with clamped edges. Since the flexural rigidities for the system at hand are comparable to those in Bennett and Eisley's work, the constant term is also neglected here. Hence, with the assumption that the beam responds with the load frequency, it is possible to write

$$
\begin{aligned}
& \tau(t)=\cos \omega t, \\
& T(t)=\cos \omega(t-\alpha), \\
& q(t)=H \cos \omega t+R \sin \omega t,
\end{aligned}
$$

where $\alpha$ is a phase angle, and the amplitude $F$ of the forcing function is given by

$$
F^{2}=H^{2}+R^{2} \text {. }
$$

Substituting equations (42), (43), and (44), into equation (39), and equating the coefficients of $\sin \omega t$ and $\cos \omega t$, gives the phase angle

$$
\omega \alpha=\tan ^{-1}\left\{-\bar{K} B \bar{a} \omega /\left[K(B \bar{a}-K \bar{b})-\bar{K}^{2} b \omega^{2}\right]\right\}
$$

and the amplitude ratio

$$
\Psi_{1} / W=2 \bar{b} K / l[(B \bar{a}-K \bar{b}) \cos \omega \alpha-\bar{K} b \omega \sin \omega \alpha] .
$$

Substituting equations (42), (43), and (44), into equation (40), and using the method of harmonic balance (see Hayashi [16]), gives, after some algebraic manipulations,

$$
\begin{aligned}
& \frac{2}{16} B_{1}^{2} W^{6}+\frac{3}{2} \bar{B}_{1}\left(\alpha_{1}-\omega^{2}+\alpha_{2} \cos \omega \alpha+\gamma_{2} \omega \sin \omega \alpha\right) W^{4}+ \\
& \quad+\left\{\omega^{4}-\left[2 \gamma_{2} \sin \omega \alpha\right] \omega^{3}+\left[\gamma_{1}^{2}+\gamma_{2}^{2}-2 \alpha_{1}+2\left(\gamma_{1} \gamma_{2}-\alpha_{2}\right) \cos \omega \alpha\right] \omega^{2}\right. \\
& \left.\quad \times\left[2\left(\alpha_{1} \gamma_{2}-\alpha_{2} \gamma_{1}\right) \sin \omega \alpha\right] \omega+\alpha_{1}^{2}+\alpha_{2}^{2}+2 \alpha_{1} \alpha_{2} \cos \omega \alpha\right\} W^{2} \\
& -\bar{C}_{1}^{2} F^{2}=0 .
\end{aligned}
$$


Once the shear coefficient $k^{2}$ and the complex moduli have been determined, and once the load amplitude $F$ has been specified, equations (46), (47), and (48) can be solved numerically to obtain $W$ as a function of $\omega$. Equation (47) can then be used to determine $\Psi_{1}$.

Another important aspect of a theory is its predictions about the resonant frequencies. The fundamental linear resonant frequency can be obtained from the frequency-amplitude plots, as given by equations (46), (47), and (48) by noting the frequency at which resonance occurs for very low values of $F$.

Still another item that warrants investigation is the stability of the motions given by equations (35), (36), (42), and (43), with $W, \Psi_{1}$, and $\omega$ as determined by equations (46), (47) and (48). To study this, small perturbations, $\delta_{1}$ and $\delta_{2}$ about the motions, defined by

$$
\begin{aligned}
\tau & =\cos \omega t+\delta_{1}(t), \\
T & =\cos \omega(t-\alpha)+\delta_{2}(t),
\end{aligned}
$$

are considered. When equations (49) and (50) are substituted into equations (39) and (40), and higher order terms in $\delta_{1}$ and $\delta_{2}$ neglected,

$$
C_{3} \check{\delta}_{1}+C_{2} \delta_{1}+\left(C_{1}+E_{1} \cos 2 \omega t\right) \delta_{1}+\left(C_{0}+E_{0} \cos 2 \omega t+F_{0} \sin 2 \omega t\right) \delta_{1}=0,
$$

where

$$
\begin{aligned}
& C_{3}=A_{2}, \quad C_{2}=-\gamma_{2} A_{1}+A_{2} \gamma_{1}+B_{2}, \\
& C_{1}=-\gamma_{2} B_{1}-\alpha_{2} A_{1}+A_{2} \alpha_{1}+(3 \beta / 2) A_{2}+B_{2} \gamma_{1}, \\
& E_{1}=(3 \beta / 2) A_{2}, \quad C_{0}=-\alpha_{2} B_{1}+B_{2} \alpha_{1}+(3 \beta / 2) B_{2}, \\
& E_{0}=(3 \beta / 2) B_{2}, \quad F_{0}=-3 \beta \omega A_{2} .
\end{aligned}
$$

With reference to the original equations in $\delta_{1}$ and $\delta_{2}$, the nature of $\delta_{2}$ can be determined, if $\delta_{1}$ is known.

In the absence of damping, equation (51) reduces to a Mathieu equation and the stability analysis follows readily (see Hayashi [16]). Here damping is not negligible, and equation (51) must be treated in full. However, the various analytic schemes tried by the authors proved to be inconclusive. It was felt that sufficient accuracy could be obtained by studying equation (51) on an analog computer, rather than by pursuing the matter further analytically. The result of that analysis will be discussed later.

\section{EXPERIMENTAL PROCEDURE}

Previous experiments involving soft elastomer cores generally reported sizable discrepancies between experiment and theory $[13,14,15]$. The present experiment was intended to show the feasibility of accurately constructing and testing sandwich beams with soft viscoelastic cores, to provide a clear-cut case for comparison with theory, and to bring out any new physical phenomena which might not have been predicted by theory. Particular attention was paid to the interface bonds, the accurate determination of the complex moduli as functions of both frequency and temperature, and the reproduction of the theoretical boundary conditions.

Primary decisions involved the geometric scale of the experiment, the type of forcing function to be applied, the type of transducer to measure the motion of the beam, and the choice of materials for the beam.

The general scale of the experiment was set by the availability of a testing machine which had been previously used by Bennett and Eisley [6] for uniform beams (see Figure 2). This set the unsupported span at $10 \mathrm{in} .(25.4 \mathrm{~cm})$. A width of $1 \mathrm{in} .(25.4 \mathrm{~mm})$ was arbitrarily taken. Electromagnetic forcing of the beam was chosen, allowing the beam to be excited with no 
mechanical contact with the exciting mechanism. This required, however, that the face sheets of the beam be made of a ferromagnetic material. An inductance transducer was chosen for measuring displacements; thus electrically conducting face sheets were required.

Both steel shim stock and silicon-iron were used for face sheets, with silicon-iron possessing better magnetic properties. The steel shim stock was available in many thicknesses, but the silicon-iron was available only in 0.002 in $(0.051 \mathrm{~mm})$ thickness.

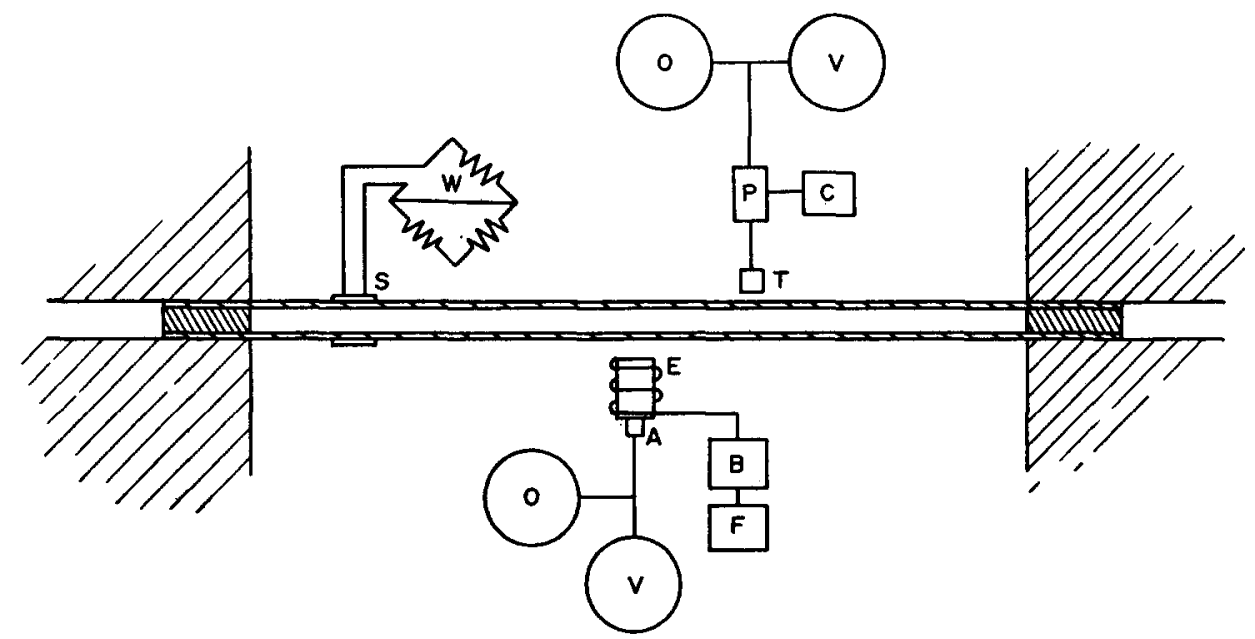

Figure 2. Test schematic. $\mathrm{A}=$ accelerometer, $\mathrm{B}=75 \mathrm{~W}$ audio amplifier, $\mathrm{C}=$ power supply, $\mathrm{E}=$ electromagnet, $F=$ function generator, $\mathrm{O}=$ oscilloscope, $\mathrm{P}=$ proximeter, $\mathrm{S}=$ strain gage, $\mathrm{T}=$ transducer, $\mathrm{V}=$ voltmeter, $\mathrm{W}=$ Wheatstone bridge.

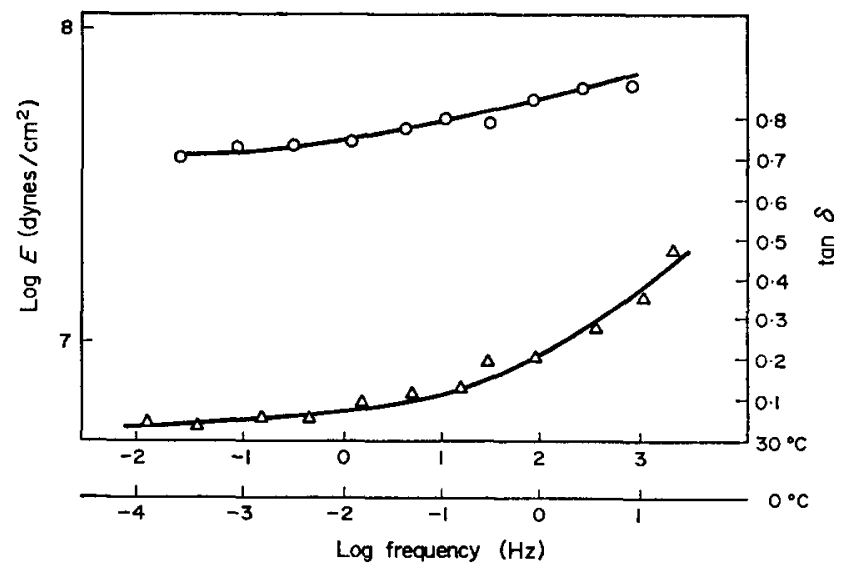

Figure 3. Complex moduli for 0.0625 in $(1.588 \mathrm{~mm})$ low durometer neoprene. $\circ-0, \log E ; \Delta \longrightarrow \Delta$, $\tan \delta$.

Four rubber-like materials were initially chosen as core materials: Solithane, nitrile, neoprene (low durometer) and neoprene (high durometer). Samples of each core material were sent to the B. F. Goodrich Research Center, where the storage and damping moduli were determined as functions of frequency and temperature. The complex moduli of the nitrile and the two neoprenes were found to be relatively stable over the desired frequency and temperature range. A typical result is shown in Figure 3. The Solithane was very erratic and was not used subsequently.

The bonding agent was required to bond rubber to steel and silicon-iron, form a thin bonding layer, and be resilient under cyclic loading. Eastman 910 cement was used and sample 
specimens were sent to the Eastman Chemical Division of the Eastman-Kodak Corporation, where the bonds formed were inspected and reported to be of excellent quality.

Since clamped-clamped boundary conditions were to be used, care had to be exercized so as not to crush the soft core in the support. As was done previously by Nicholas and Heller [13], stiff aluminum fillers were used to replace the soft core for that portion of the beam clamped in the support.

Approximately 30 different specimens were constructed in which various combinations of core and face sheet thicknesses were tried. Magnetic saturation at times limited the amplitude of the forcing function which could be induced and it was difficult to excite large amplitudes in the stiffer beams. The specimens with 0.0625 in $(1.588 \mathrm{~mm})$ low durometer neoprene cores and $0.002-0.010$ in $(0.051-0.254 \mathrm{~mm})$ faces were found to be the best.

The test rig was basically a lathe bed. Clamping was accomplished by inserting the ends of the beam between large steel blocks and then bolting the blocks, specimen and lathe bed together. One end of the clamping arrangement was moveable and after specimens were mounted in the supports it was possible to eliminate extraneous axial loads. To accomplish this, strain gages were mounted on opposite sides of the beam and the clamps were adjusted until no axial load was imposed on the beam.

The electromagnet, which was made in an $E$ shape, was driven by a sinusoidal function generator of frequency $\omega_{\mathrm{E}}$, and a $75 \mathrm{~W}$ amplifier. The resulting force, $F_{\mathrm{f}}$, between the face sheets and the electromagnet, was perpendicular to the face sheets and was proportional to the square of the current in the electromagnet, i.e.

$$
F_{\mathrm{f}} \sim 1+\cos \omega t
$$

where $\omega=2 \omega_{\mathrm{E}}$. Thus the exciting force had twice the frequency of the function generator. As mentioned before, the constant term in equation (52) is not considered in the development of the theoretical results. Also, the dimensions of the electromagnet were such that the spatial distribution of the forcing function approximated equation (41). The magnitude $F$ of the forcing function was determined as follows. The acceleration of the magnet, which was suspended by wires and free to move in a horizontal direction, was determined from an attached accelerometer. Then, noting the equal and opposite forces on magnet and beam, $F$ was obtained since the mass of the magnet was known.

A Bentley-Nevada inductive transducer was used to measure displacements. It was essentially a small coil excited by a $2 \mathrm{MHz}$ signal. When placed near an electrically conducting body, the coil generates eddy currents in the body, the electromagnetic field is changed, and a voltage is generated which is proportional to the distance between the coil and the body. A linear range of $0.14 \mathrm{in}$. $(3.56 \mathrm{~mm})$ was obtained for the particular transducer used, and this was sufficient for the tests. Two such transducers were actually used and if their position along the length of the beam was varied, it was possible to obtain the mode shape.

The beams proved to be heavily damped and so the natural frequencies could not be readily determined by observing the period of free oscillations. The natural frequencies were obtained from a frequency-response approach, using low values of the forcing amplitude $F$, with the resonant frequency approximating the natural frequency.

\section{EXPERIMENTAL RESULTS AND COMPARISON WITH THEORY}

Shown in columns 5 and 6 of Table 1 are the theoretical and experimental natural frequencies for the eight specimens tested most thoroughly. Theoretical values were obtained by solving equation (48) for small values of $F$. As mentioned previously, they were also obtained by solving the linear, undamped, eigenvalue problem (see Nicholas and Heller [13]). The two 


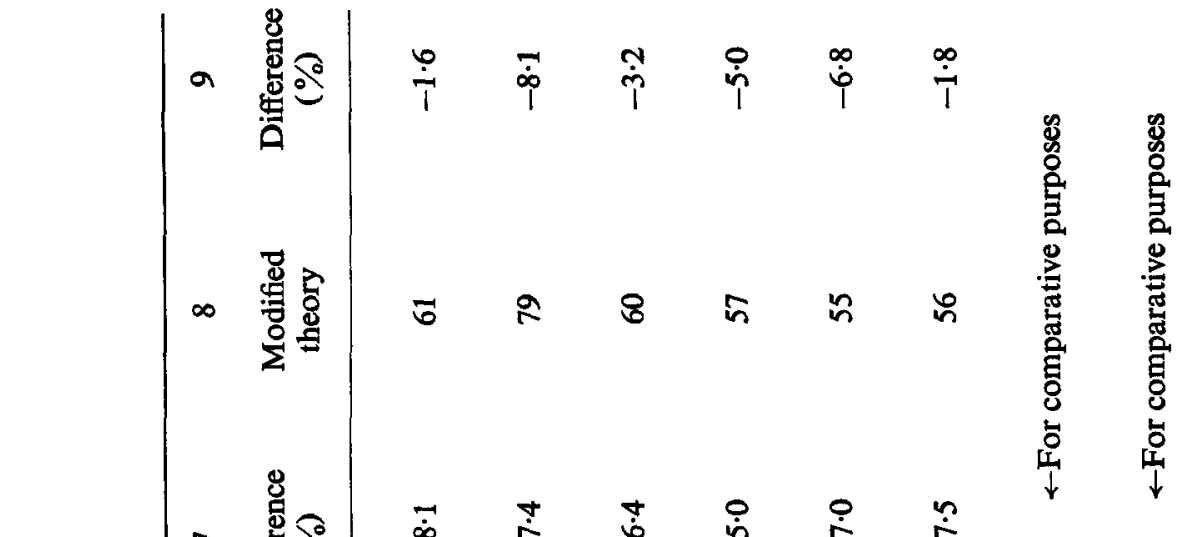

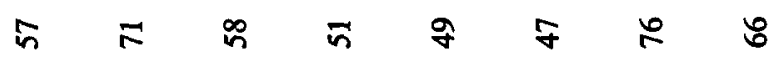

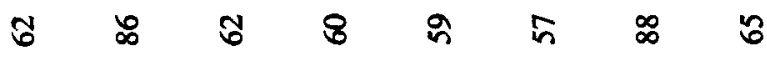

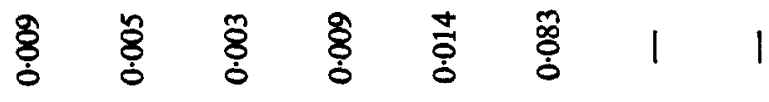

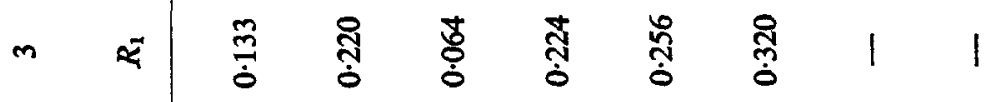

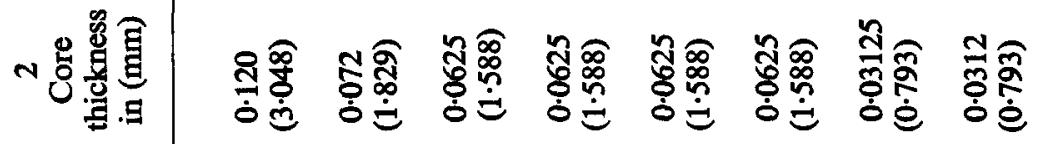

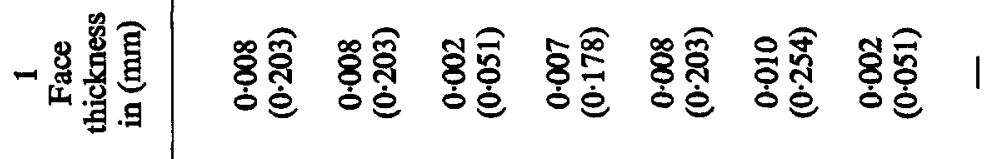

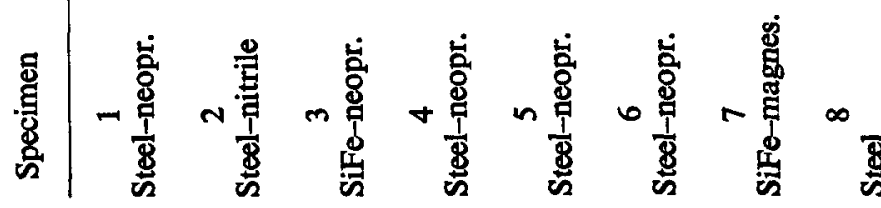


approaches yielded almost identical results, which gave confidence in approximation of the natural frequencies by resonant frequencies.

It is seen that the predictions of the simplified theory are low by $6-17 \%$. This feature is somewhat unusual as difficulties in experimental reproduction of the theoretical conditions often result in theoretical frequencies being higher than experimental ones. It should be noted that Jones, Salerno and Savacchio [14] also reported that experimental predictions were consistently higher than theoretical predictions. Recall that for the simplified theory to be accurate, the inequalities, equations (30) and (31), should be satisfied, at least for infinite, undamped beams. Shown in columns 3 and 4 of Table 1 are the pertinent ratios and it is seen that the specimens used are marginal in satisfying the requirement that $R_{1} \ll 1$.

If it is assumed that the improved estimate due to $\mathrm{Yu}$ [9], as given by equation (32), which is for infinite undamped beams, applies here also, a modified simplified theory results, and its predictions are shown in column 8 of Table 1 . It is seen that the modified theory agrees well with experiment, the discrepancies between the two running from 2 to $8 \%$. There was a natural conflict in the design of the experiment between the need of the simplified theory for thin face sheets and the need of the experiment for thick face sheets for adequate magnetic attraction. The use of the modified simplified theory seems to bridge this gap and to give confidence that the simplified theory should be adequate for many practical situations involving truly thin face sheets.

Frequency-amplitude relations were studied most thoroughly for specimens 3 and 5 , beams which were best suited to the test equipment. Typical theoretical and experimental results are shown in Figures 4 and 5 . When plotted against normalized frequency $\omega / \omega_{0}$, where $\omega_{0}$ is the natural frequency, the experimental amplitude shows good agreement with the theoretically obtained curves. The general shapes of the curves are similar with regard to non-linear hardening and peak amplitudes, the experimental peak amplitudes being slightly higher than their theoretical predictions. Another common occurrence was the appearance, experimentally, of a slight peak at about one half of the linear resonant frequency. These peaks, which stem from a superharmonic response, are discussed later. The deviation of the theoretical curve just before resonance can be attributed in part to the effect of the axial (in-plane) elasticity of the supports, a problem also encountered by Bennett and Eisley [6] in their study of homogeneous beams. When lower amplitude forcing functions were applied to these specimens, it was found that the discrepancy was less, implying that it is due to a nonlinear effect, such as a reduction of in-plane support rigidity. One suspects in-plane stiffness rather than rotational stiffness of the supports because small amplitude motion is not affected. Lack of perfect axial rigidity at the supports means that the beam does not stiffen with amplitude as much as with a perfect support, causing the experimental resonant response curve to incline to the left of the perfectly rigid system, and allowing greater amplitudes than the perfect system.

A check on the validity of the single space mode assumption was also made. As is seen from Figure 6, which gives a typical result, the measured mode shape agrees quite well with the theoretical one given by equation (37). An exception is the special case when the forcing frequency is equal to one half the linear resonant frequency. This latter circumstance produced the only detectable deviation from the assumed mode shape. As mentioned previously, there is a difference between the theoretical and experimental results for amplitude of motion in the vicinity of this frequency. Figure 7 shows the magnitude of the response of the beam as a function of $x$. Two transducers placed equidistant from the center of the beam established the spatial symmetry of the motion. The Fourier components of the time response were determined using a Quan-Tech model 304 waveform analyzer, an instrument with a resolution bandwidth of $2 \mathrm{~Hz}$. The signal at a given $x$ station was found to be the sum of a response with a frequency the same as that of the forcing function plus a superharmonic response at twice the 


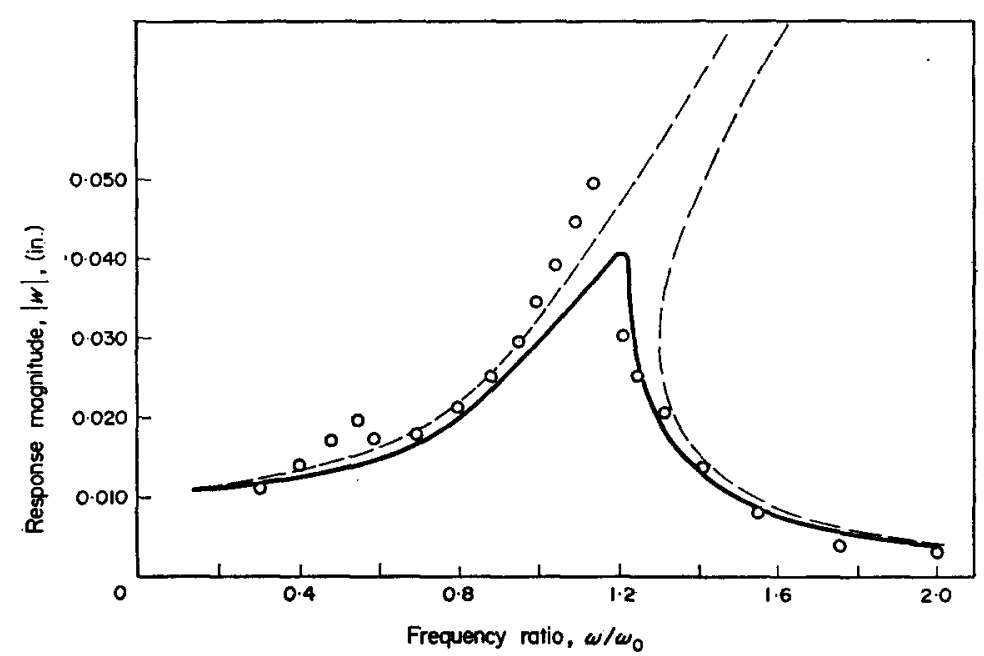

Figure 4. Frequency response at midspan. Specimen 3. Face sheets $=0.002$ in $(0.051 \mathrm{~mm}) \mathrm{Si}-\mathrm{Fe}$; core $=$ 0.0625 in $(1.588 \mathrm{~mm})$ low durometer neoprene. $F=0.085 \mathrm{lbf}(0.378 \mathrm{~N}) .---$, theory (undamped); - , theory (damped); 00000 , experiment.

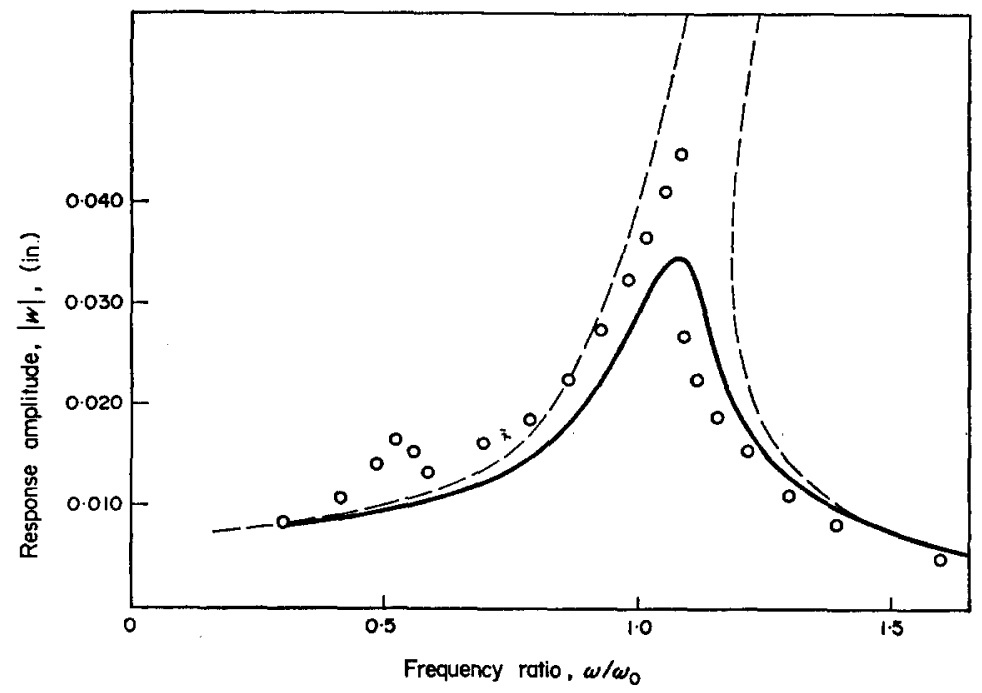

Figure 5. Frequency response at midspan. Specimen 5 . Face sheets $=0.008$ in $(0.203 \mathrm{~mm})$ steel; core $=$ 0.0625 in $(1.588 \mathrm{~mm})$ low durometer neoprene. $\mathrm{F}=0.170 \mathrm{lbf}(0.756 \mathrm{~N}) .--$, theory (undamped); theory (damped); 00000 , experiment.

forcing frequency (the linear resonant frequency). A general functional form for this type of motion might be proposed:

$$
w(x, t)=W_{1}(x) \sin \omega_{1} t+W_{2}(x) \sin \left(2 \omega_{1} t+\phi(x)\right) .
$$

A thorough investigation of $W_{1}(x), W_{2}(x)$, and $\phi(x)$ should be carried out for a full understanding of the details of the motion, a study felt to be beyond the scope of the present paper.

The results of a typical stability analysis are shown in Figure 8. The instability zones for the undamped case are given by the curves emanating from the frequency axis. These were obtained from the stability chart of Mathieu's equation. The damped problem was treated by analyzing equation (51) on an analog computer (Applied Dynamics model AD-1-64PB), using the value of the forcing frequency in the determination of the coefficients. With this 
assumption it was found that the motion given by equations (35), (36), (42), (43), (44), (46), (47) and (48) is stable for the moderate forcing function amplitudes that were experimentally used. However, the whole question of superharmonic response and stability should be regarded as an open one requiring further work. The frequency assumption of equations (42) and (43) and the single space mode assumption of equation (37) should be altered. Of great importance is the development of an approach for handling damping when the beam response is at more than one frequency.

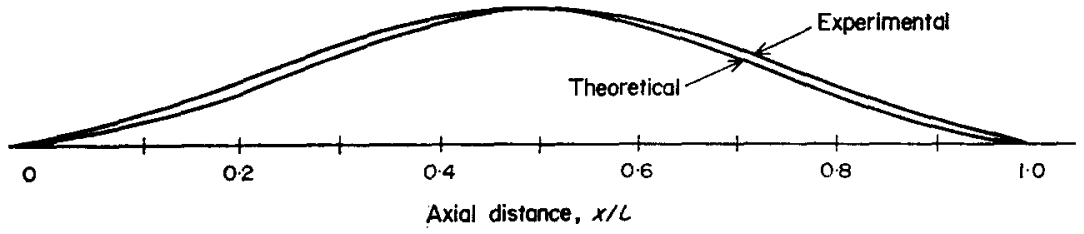

Figure 6, Mode shape $\left(\omega=\omega_{0}\right)$. Specimen 5.

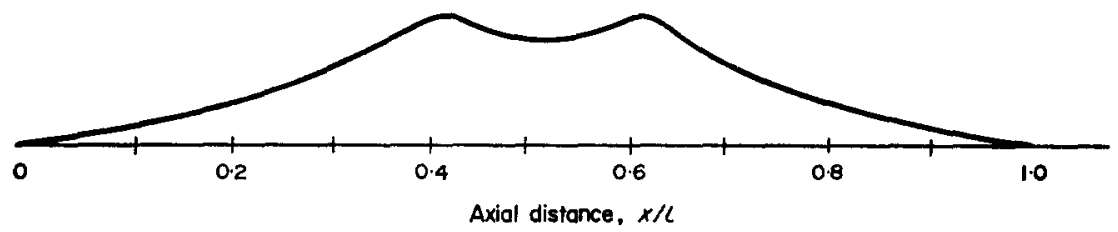

Figure 7. Mode shape during superharmonic response $\left(\omega=\omega_{0} / 2\right)$. Specimen 5 .

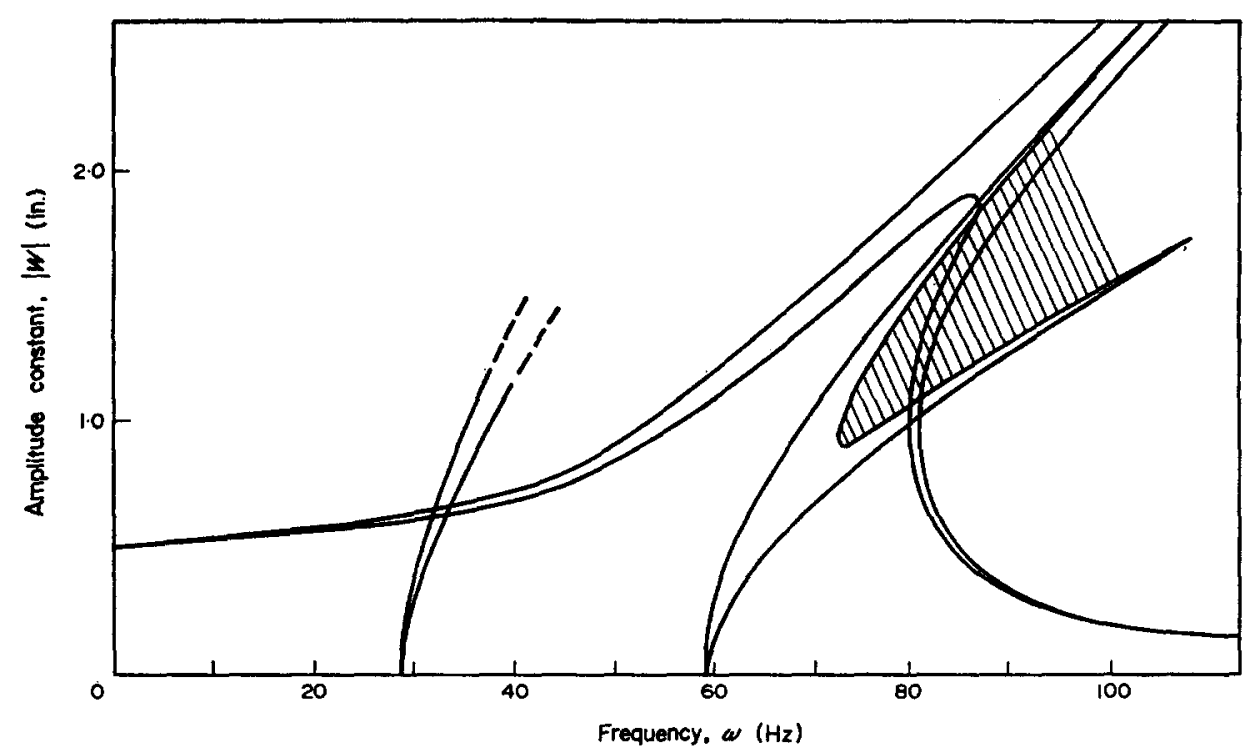

Figure 8. Damped stability zones (compared to undamped). Specimen 3. Forcing function amplitude $=0.34$ lbf (1-512 N). Shaded zones give unstable motions.

\section{ACKNOWLEDGMENTS}

The authors are indebted to Dr N. Trivisonno of the B. F. Goodrich Research Center for his determination of the complex moduli and to Mr W. F. Thomsen of the Eastman-Chemical Division of the Eastman-Kodak Corporation for inspecting the bonds. They are also indebted to Dr J. Bennett who developed the initial apparatus. Financial support is gratefully acknowledged by the first author for a National Science Foundation Summer Research Grant. Also, the work was funded in part by National Science Foundation Grant No. GK-10725. 


\section{REFERENCES}

1. J. G. EISLEY 1966 Nonlinear Deformation of Elastic Beams, Rings, and Strings. Applied Mechanics Surveys. Washington, D.C.: Spartan Books.

2. S. R. WOODALL 1966 Int. J. nonlinear Mech. 1, 217. On the large amplitude oscillations of a thin elastic beam.

3. A. V. SRINIVASAN 1966 Int.J. nonlinear Mech. 1, 179. Nonlinear vibration of beams and plates.

4. D. A. Evensen 1968 AIAA J. 6, 370. Nonlinear vibrations of beams with various boundary conditions.

5. W. Y. TSENG 1969 AFOSR scient. Rep. ASRL-TR-159-1. Nonlinear vibrations of straight and buckled beams under harmonic excitation.

6. J. A. BenNetT and J. G. EISLEY 1970 AIAA J. 8, 734. A multiple degree-of-freedom approach to nonlinear beam vibrations.

7. J. E. Ruzika (Ed.) 1959 Structural Damping. New York: ASME.

8. Y. Y. YU $1960 \mathrm{~J}$. Aerospace Sci. 27, 272. Flexural vibrations of elastic sandwich plates.

9. Y. Y. Yu 1960 J. Aerospace Sci. 27, 894. Simplified vibration analysis of elastic sandwich plates.

10. Y. Y. Yu 1962 J. Aerospace Sci. 29, 790. Damping of flexural vibration of sandwich plates.

11. Y. Y. YU 1962 J. acoust. Soc. Am. 34, 1176. Nonlinear flexural vibrations of sandwich plates.

12. Y. Y. Yu and J. L. LaI 1966 J. appl. Mech. 33, 934. Influence of transverse shear and edge condition on nonlinear vibrations of sandwich plates.

13. T. Nicholas and R. A. Heller 1967 J. exp. Mech. 9, 110. Determination of the complex shear modulus of a filled elastomer from a vibrating sandwich beam.

14. J. W. Jones, V. L. SAlerno, and A. Savacchio 1967 J. Engng. Ind. 89, 438. An analytical and experimental evaluation of the damping capacity of sandwich beams with viscoelastic cores.

15. J. A. AGbasier and P. Grootenhuis $1968 \mathrm{~J}$. mech. Engng. Sci. 10, 269. Flexural vibration of symmetrical multi-layer beams with viscoelastic damping.

16. C. HAYASHI 1964 Nonlinear Oscillations in Physical Systems. New York: McGraw-Hill. 